\title{
Deformation Design Method Based on SBF Model
}

\author{
https://doi. org/10.3991/ijoe. v14i03. 8417 \\ Qinzhou Niu( $\left.{ }^{(}\right)$, Yi Liu \\ Guilin University of Technology, Guilin, China \\ niuqinzhou@ 126 . com
}

\begin{abstract}
The structure-behavior-function model is a kind of concept design model and we apply it to the deformation design mainly to express the design concept and design process. In this paper, the SBF model is used to express a complete design experience knowledge and is used as a node for design reasoning. We also add the non-axiomatic logic and a calculation algorithm of semantic similarity to the SBF model to accomplish the process of deformation design. Finally, turbine blade is taken as an example to prove that the method can realize the deformation designing of parts. Meanwhile, non-axiomatic logic which is able to reduce the repetitive work of the designers is an effective way to simplify the designing.
\end{abstract}

Keywords-structure-behavior-function, non-axiomatic logic, deformation design

\section{Introduction}

The deformation design is a comprehensive practical design method which extracts the main variable parameters from the local feature structure, as well as modifies its values of dimension, with the preservation of the basic functions of the product and a small change of the working principle, in order to meet the different needs of the users, and improve the response speed for the market. Deformation design is divided into two types i.e. the deformed of the geometric dimensions and the restructuring deformed of the overall structure. The deformation of the geometric dimensions is the change of the parameters of individual parts, but the structural characteristics of the product is not affected. But based on the individual needs of customers, the restructuring deformed of the overall structure changes the type of parts or the number of components. This deformation will usually change overall structure. Therefore, the deformation design includes both the conceptual design and the detailed design.

The SBF model was developed on the basis of FR[1-2]. In SBF, the structure refers to the components, substances contained in the components, and the relationship between components. Function can be expressed in the form of a schema, which specifies the preconditions and the postconditions of the function. Behavior represents a series of states and the transition between them, and the states and the process of transition can also be represented by schema. In this paper, we extend the SBF model to represent the information of structure, behavior, and function in the designing. 
Through external functions, an extended SBF model allows the designer to master the objects which are associated with the designed part in various life cycle.

The combination of artificial intelligence and design theory is a direction of design theory. The non-axiomatic logic(NAL)[3-4]which is an intelligent reasoning method can be applied to conceptual design and innovative design. A brief introduction of it is presented here. Non-axiom logic is a term logic and it has the following characteristics: 1)Term logic is a kind of syllogistic reasoning. Each proposition is in the form of the "subject, copula, predicate". The term here refers to a certain concept that can be represented by the name of the concept, while the copula can be interpreted as "is" or "associated with". 2)The specific reasoning form is that two propositions that have a term in common are known, which can be used to produce the third proposition (i. e. the conclusion).

The remainder of the paper is organized as follows. In section two, we introduce the SBF ontology model which is used to indicate design knowledge. Section three introduces non-axiomatic logic model of the deformation designing. The fourth section describes the semantic calculation of deformation design reasoning. In section five, through an examples, the concrete realization of the deformation design theory proposed in this paper is discussed. Section six introduces a deformed design system. Section seven is conclusion.

\section{Model of SBF Ontology Representation of Deformed Design Knowledge}

In this paper, SBF theory is used as a model of ontology representation of the knowledge . It is mainly used to refine the term in non-axiomatic logic, and divide each term into three levels i. e. structure, behavior and function. The SBF model is the precondition of quantitative calculation of semantic similarity. And the original SBF model is an experience-grounded model, but when we unfold the terms according to the SBF model, then calculate the similarity, we incorporate the SBF model with a process of quantitative calculation. This process itself is a development of this model. At the same time, the SBF model also has important significance in performing the deformation. Since each term is divided into three levels, then deformed of the three levels should all be taken into account. We think that, for example, between two items, function, behavior is similar and can be transformed, then we can assume that the structure can also achieve the transformation. That is to say we do not necessarily take into account all the three terms in each design. We can only consider one or two of them, then a new concept can be constructed. Whether this concept is feasible or not, designers need to further think about it. In order to express the design knowledge in detail, we also extended the SBF model[5], the specific representation is as follows. 


\subsection{SBF model}

Structure. Structure refers to the appearance of the product, and is divided into composition representation and form representation. The composition representation describes what parts are included in the product and their parameters. Each part in the composition representation can be illustrated by $<$ name, parameters $>$. Name describes the name of the part, and the parameter is the physical attribute. In order to support the detailed design, the original SBF model is expanded to include form representation. The form representation describes the geometrical information of the part by the positional relationship parameter between the features.

Behavior. The behavior of a part refers to the change of its own state in its life cycle, such as the movement of the workbench. The original SBF model only considers the behavior that intend to achieve the desired function, but the extended SBF model also includes the behavior of a part in a variety of life cycles such as machining. Another difference is that the extended SBF model uses quantitative methods to represent the degree of change in state. The behavior can be represented quantitatively by four elements <name, parts, parameters, causes $>$. Name represents the name of a behavior. The part refers to which part the behavior belongs to, and the parameter intend to quantify the change of state. Cause is a object which stimulates the current behavior.

Function. The traditional SBF model only considers the relationship between input and output. In extended SBF model, the function is defined as an expected relationship between a part (subject) and another part (object) in any life cycle. The concept of function here is more like affordance.

Function can be divided into internal functions and external functions. An internal function means that the subject and the object belong to one product, and the internal functions can be further divided into static and dynamic functions. In static functions, objects are often treated as static parts. For example, the workbench has the function of carrying the workpiece. The workpiece can be seen as a static object. A static function can be expressed as three elements $<$ subject, verb, parameters $>$. The verb is used to describe the functional relationship. Dynamic function is concerned with the change of dynamic behavior of the object, and a dynamic function can be expressed with three elements $<$ subject, verb, parameters $>$.

An external function means that its object is not a part of the product. The external function which is similar to the internal function can be expressed by four elements $<$ subject, verb, external object, parameters $>$. Compared to existing CAD system life cycle management tools, adding external features has a major advantage. With function information, external objects in the various life cycles can be taken into account, so it helps designers to understand the detailed design process of the product better. For example, various tools (fixtures, mills, etc. ) for machining and assembling parts can also be reflected in external functions, which will make it easier for designers to understand how the tools affect the results of detailed design. 


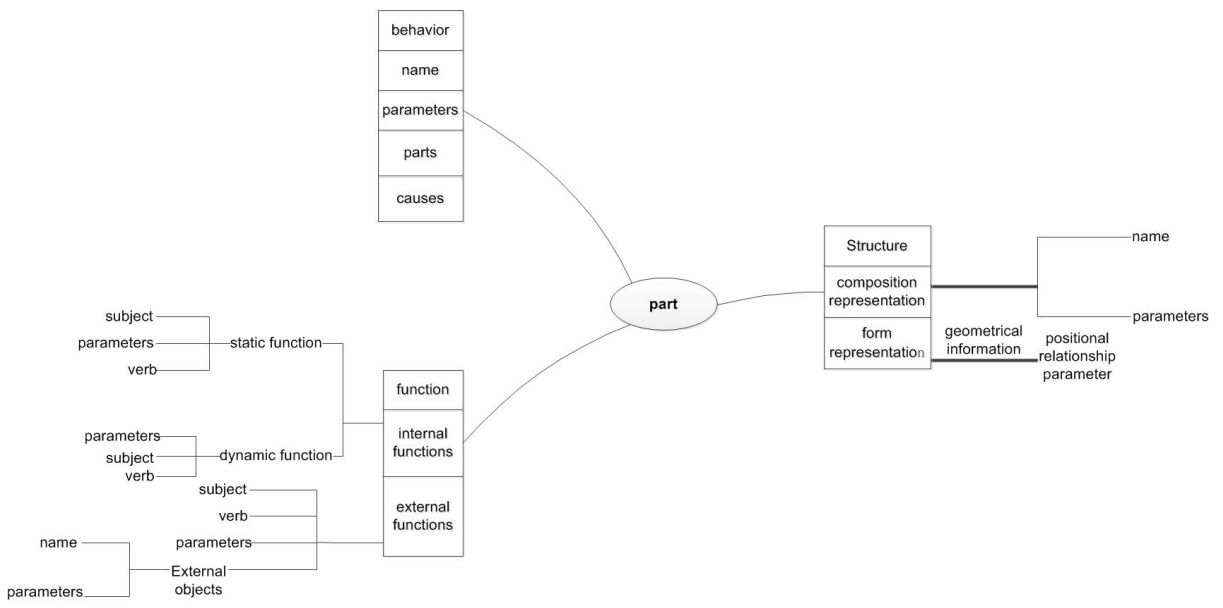

Fig. 1. An structure-behavior-function model

\section{$3 \quad$ Non-axiomatic Logic Modeling of Deformation Design Process}

In this paper, select reasoning rule of NAL as the main reasoning form of the deformation design, because NAL provides us with not only deduction, induction, abduction and other reasoning methods but their formula of true value. These can completely meet our needs in the deformation design. Another important feature of NAL which distinguishes it from other reasoning systems is that reasoning can be done with insufficient knowledge and resources. When it is used to answer questions raised by existing knowledge, these knowledge can be uncertain and incomplete. So every knowledge in the system is only correct to some extent, and each piece of knowledge can be revised according to new knowledge[3]. On the basis of the original NAL model, we have not only improved its terms, but also introduced an algorithm for confidence c. Because the original non-axiomatic logic does not explicitly put forward the algorithm of confidence, it only estimates confidence empirically. After the addition of the computational model, the accuracy of the true value which base on both a quantitative calculations and a empirical estimate is greatly increased. We use the true value to assess the deformation process. A deformation with a higher true value represents a higher degree of reliability, and a deformation only with a lower true value represents that the deformation requires further verification. For the sake of convenience, we use nodes to stand for a design implementation of the object.

\subsection{A Brief Introduction to Non-axiomatic Logic}

We have described the internal structure of a part (term), we will show the relationship between two parts (terms) in this section. In the non-axiomatic logical lan- 
guage, the general proposition is composed of subject, predicate, the inheritance relation, and the true value:

$$
\mathrm{S} \subset \mathrm{P}<\mathrm{f}, \mathrm{c}>
$$

Where $\mathrm{S}$ denotes the subject. $\mathrm{P}$ denotes the predicate. $\subset$ represents the inheritance, and $<\mathrm{f}, \mathrm{c}>$ denotes the true value of the proposition. The proposition indicates that $\mathrm{S}$ is the specificity of $\mathrm{P}$ and $\mathrm{P}$ is the generalization of $\mathrm{S}$. True value of the proposition is represented by a number pairs $\langle f, c\rangle$. $f$ is the frequency that represents the proportion of successful evidence in all available evidence. $\mathrm{c}$ is the confidence, considering the evidence that the system has now and in the future. Proposition with true value is the complete reasoning, and the proposition without true value can only be a logical guess. NAL is described as an inheritance relationship. We can treat each term as a node, and the proposition is the concrete relationship between the two nodes, while corresponding truth value is the strength of the relationship. The reasoning in the logic is expressed in form of a syllogism. A pair of statements are given. If they share a common term, then the conclusion between the other two terms can be derived from the reasoning.

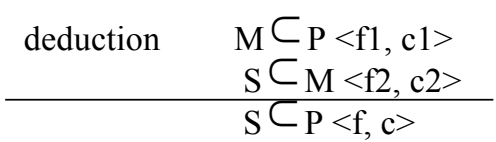$$
\text { abduction } \mathrm{P} \subset \mathrm{M}<\mathrm{fl}, \mathrm{cl}>
$$$$
\mathrm{S} \subset \mathrm{M}<\mathrm{f} 2, \mathrm{c} 2>
$$

Induction $\mathrm{M} \subset \mathrm{P}<\mathrm{fl}, \mathrm{c} 1>$

$\mathrm{M} \subset \mathrm{S}<\mathrm{f} 2, \mathrm{c} 2>$

$$
\mathrm{S} \subset \mathrm{P}<\mathrm{f}, \mathrm{c}>
$$

Table 1. A summary of basic syllogistic rules

\begin{tabular}{cccc}
\hline Inference type & function & frequency & confidence \\
\hline deduction & Fded & $\mathrm{f}=\mathrm{f} 1 \mathrm{f} 2$ & $\mathrm{c}=\mathrm{f} 1 \mathrm{c} 1 \mathrm{f} 2 \mathrm{c} 2$ \\
abduction & Fabd & $\mathrm{f}=\mathrm{f} 2$ & $c=\frac{f_{1} c 1 c 2}{f_{1} c 1 c 2+k}$ \\
induction & Find & $\mathrm{f}=\mathrm{f} 1$ & $c=\frac{f_{2} c_{1} c 2}{f_{2} c_{1} c 2+k}$ \\
\hline
\end{tabular}

In deductive reasoning, one of the common term is a subject and the other is a predicate. In the abduction reasoning, the common term is two predicates. In the inductive reasoning, the common term is two subjects. If only the combination of the premise is considered, then the three kinds of reasoning have exhausted all the possibility. The following passage will show how to apply non-axiomatic logic to the deformation design. 


\section{Semantic Computation of Deformed Design Reasoning}

\subsection{Calculation method of $f$}

Frequency f:f is the proportion of the successful deformed. NAL has an experience-grounded semantics. That is to say the determination of frequency needs to base on multiple experiments. In fact, a lot of design is difficult to obtain the existing experimental data, because the design work itself may be entirely new. To solve this problem, for those that can not find knowledge of the design, the real frequency can be replaced by a mean value which is estimated by a certain number of experts.

\subsection{The calculation algorithm of confidence $c$}

Ontology coding. In order to complete the deformation between the two CAD models, we describe two different concepts with the product semantic expression language (PSRL) to determine whether two different CAD models are semantically similar[6]. The advantage of this approach is that the semantics of the CAD model can be clearly expressed. This can help us to compare the semantic similarities of these models. Note that the ontology here refers to the concept of the term mentioned above. For example, suppose that the 11th stage rotational blade of a turbine is a data ontology, and the sub-last one is another data ontology. The PSRL for the functions of the two ontology is described below.

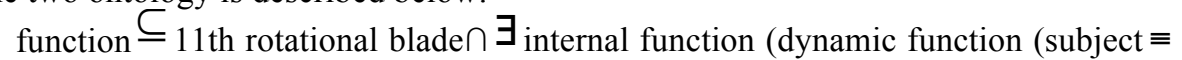
rotational bladeUverb $\equiv$ rotate Ufunctional parameter $\equiv 10000 \mathrm{rpm}$ ) Ustatic function (subject $\equiv$ rotational blade $u$ functional relation $\equiv$ reaction degree $U$ functional parameter $\equiv 0.23)$ ) $\cap=\exists$ external function (subject $\equiv$ rotational bladeUverb(efficiencyUrelative velocity) Ufunctional parameter (efficiency $\equiv 0.9085 \cup$ relative velocity $\equiv 360 \mathrm{~m} / \mathrm{s}$ ) Uexternal objects (name $\equiv$ steam))

function $\complement_{\text {sub-last stage rotational blade } \cap} \exists_{\text {internal function (dynamic function }}$ (subject $\equiv$ rotational bladeUverb $\equiv$ rotate Ufunctional parameter $\equiv 10000 \mathrm{rpm}$ ) Ustatic function (subject $\equiv$ rotational blade $\cup$ functional relation $\equiv$ reaction degree $U$ functional parameter $\equiv 0.4)$ ) $\cap=\exists$ external function (subject $\equiv$ rotational blade Uverb(efficiencyUrelative velocity)Ufunctional parameter (efficiency $\equiv$ $0.8962 \cup$ relative velocity $\equiv 480 \mathrm{~m} / \mathrm{s}$ ) Uexternal objects (name $\equiv$ steam))

With the reasoning mechanism PSRL, we can see that their description is very similar. In order to achieve the transformation between the two models, we want to quantify the semantic similarity between them. In recent years, a lot of measure methods have been put forward to solve this issue. In this paper we have chosen AbdulGhafour's method[7], which is a attribute-based approach. Specifically, the semantic similarity of two concept can be aggregated as a weighed sum of the similarity of their function, behavior and structure. Let $C_{1}, C_{2}$ as a concept pair. $\operatorname{Sim}_{G}\left(C_{1}, C_{2}\right)$ is the similarity of the functions of $\mathrm{C}_{1}$ and $\mathrm{C}_{2}$. $\operatorname{Sim}_{\mathrm{X}}\left(\mathrm{C}_{1}, \mathrm{C}_{2}\right)$ is the similarity of behavior 
of $\mathrm{C}_{1}$ and $\mathrm{C}_{2} . \operatorname{Sim}_{\mathrm{J}}\left(\mathrm{C}_{1}, \mathrm{C}_{2}\right)$ is the structure similarity of $\mathrm{C}_{1}$ and $\mathrm{C}_{2}$. Then semantic similarity of $\mathrm{C}_{1}, \mathrm{C}_{2}$ can be defined as:

$\operatorname{Sim}\left(\mathrm{C}_{1}, \mathrm{C}_{2}\right)=\mathrm{w}_{1} \operatorname{Sim}_{\mathrm{G}}\left(\mathrm{C}_{1}, \mathrm{C}_{2}\right)+\mathrm{w}_{2} \operatorname{Sim}_{\mathrm{X}}\left(\mathrm{C}_{1}, \mathrm{C}_{2}\right)+\mathrm{w}_{3} \operatorname{Sim}_{\mathrm{J}}\left(\mathrm{C}_{1}, \mathrm{C}_{2}\right)$

Where $\mathrm{w}_{1}, \mathrm{w}_{2}, \mathrm{w}_{3}$ is the weights, and , $\mathrm{w}_{1}+\mathrm{w}_{2}+\mathrm{w}_{3}=1$. This paper chooses Petrakis's method which is one of the attribute-based similarity measure to calculate the contribution components $\operatorname{Sim}_{\mathrm{G}}\left(\mathrm{C}_{1}, \mathrm{C}_{2}\right), \operatorname{Sim}_{\mathrm{X}}\left(\mathrm{C}_{1}, \mathrm{C}_{2}\right)$ and $\operatorname{Sim}_{\mathrm{J}}\left(\mathrm{C}_{1}, \mathrm{C}_{2}\right)$.

$$
\text { Simsynsets/glosses }\left(\mathrm{C}_{1}, \mathrm{C}_{2}\right)=\left|\mathrm{S}\left(\mathrm{C}_{1}\right) \mathrm{S}\left(\mathrm{C}_{2}\right) / / \mathrm{S}\left(\mathrm{C}_{1}\right) \mathrm{S}\left(\mathrm{C}_{2}\right)\right|
$$

Where $\mathrm{S}\left(\mathrm{C}_{1}\right), \mathrm{S}\left(\mathrm{C}_{2}\right)$ are the attribute sets of concepts $\mathrm{C}_{1}$ and $\mathrm{C}_{2}$, respectively.

The weight of the similarity calculation. This section will describe a calculation method of weights. For the sake of generality, the $n$ contribution components $f_{1}\left(C_{1}\right.$, $\left.\mathrm{C}_{2}\right), \mathrm{f}_{2}\left(\mathrm{C}_{1}, \mathrm{C}_{2}\right) \ldots \mathrm{f}_{\mathrm{n}}\left(\mathrm{C}_{1}, \mathrm{C}_{2}\right)$ are taken into account in the similarities of $\mathrm{C}_{1}$ and $\mathrm{C}_{2}$. $\mathrm{w}_{1}, \mathrm{w}_{2}, \mathrm{w}_{3} \ldots \mathrm{w}_{\mathrm{n}}$ are the weights of $\mathrm{f}_{1}\left(\mathrm{C}_{1}, \mathrm{C}_{2}\right), \mathrm{f}_{2}\left(\mathrm{C}_{1}, \mathrm{C}_{2}\right) \ldots \mathrm{f}_{\mathrm{n}}\left(\mathrm{C}_{1}, \mathrm{C}_{2}\right)$ respectively. And $0 \leq w_{1}, w_{2}, w_{3} \ldots w_{n} \leq 1, \mathrm{w} 1+\mathrm{w} 2+\mathrm{w} 3 \ldots \ldots \mathrm{wn}=1$. Then the overall semantic similarity can be expressed as:

$$
\operatorname{Sim}\left(\mathrm{C}_{1}, \mathrm{C}_{2}\right)=\mathrm{w}_{1} \mathrm{f}_{1}\left(\mathrm{C}_{1}, \mathrm{C}_{2}\right)+\mathrm{w}_{2} \mathrm{f}_{2}\left(\mathrm{C}_{1}, \mathrm{C}_{2}\right) \ldots+\mathrm{w}_{\mathrm{n}} \mathrm{f}_{\mathrm{n}}\left(\mathrm{C}_{1}, \mathrm{C}_{2}\right)
$$

In general, the Pearson correlation coefficient can be used to evaluate the actual similarities and calculated similarities of a certain number of sample pairs. The higher the correlation coefficient is, the better the accuracy of the calculated similarities are[8]. It is difficult to obtain the actual similarity of a sample pair, since the similarity is a kind of subjective judgment of human. The actual similarity also need to be replaced by a mean value which is estimated by a certain number of experts. According to the above description, we intend to calculate a group of weights that can maximize the Pearson correlation coefficient. The specific algorithm is as follows, let $\mathrm{N}$ be the number of concept pairs whose semantic similarity need to be measured. $A_{i}$ $\left(\mathrm{C}_{\mathrm{i} 1}, \mathrm{C}_{\mathrm{i} 2}\right)$ is the actual semantic similarity of the $\mathrm{i}$-th concept pair. $\mathrm{U}=\left[\mathrm{f}_{\mathrm{i} 1}\left(\mathrm{C}_{\mathrm{i} 1}, \mathrm{C}_{\mathrm{i} 2}\right)\right.$, $\left.\mathrm{f}_{\mathrm{i} 2}\left(\mathrm{C}_{\mathrm{i} 1}, \mathrm{C}_{\mathrm{i} 2}\right) \ldots \ldots \mathrm{f}_{\mathrm{in}}\left(\mathrm{C}_{\mathrm{i} 1}, \mathrm{C}_{\mathrm{i} 2}\right)\right]^{\mathrm{T}}$ is a vector. $\mathrm{V}=\left[\mathrm{A}_{\mathrm{i}}\left(\mathrm{C}_{\mathrm{i} 1}, \mathrm{C}_{\mathrm{i} 2}\right)\right]^{\mathrm{T}}$ is a vector. $\mathrm{W}=\left[\mathrm{w}_{1}, \mathrm{w}_{2}\right.$, $\left.\mathrm{w}_{3} \ldots \ldots \mathrm{w}_{\mathrm{n}}\right]^{\mathrm{T}}$ is also a vector. Then the Pearson correlation coefficient between actual similarity and calculated similarity can be expressed as:

$$
\operatorname{Corr}(\mathrm{WTU}, \mathrm{V})=\frac{w^{T} \operatorname{cov}(U, V)}{\sqrt{w^{T} \operatorname{cov}(U, U) w} \sqrt{\operatorname{cov}(V, V)}}=\frac{w^{T} \sum_{U V}}{\sqrt{w^{T} \sum_{U U}^{w}} \sqrt{\sum_{V V}}}
$$

Where cov is covariance, and $\operatorname{cov}(\mathrm{U}, \mathrm{U})=\Sigma \mathrm{UU}, \operatorname{cov}(\mathrm{U}, \mathrm{V})=\Sigma \mathrm{UV}, \operatorname{cov}(\mathrm{V}, \mathrm{V})=\Sigma$ $\mathrm{VV}$ are the following matrices respectively:

$\Sigma_{\mathrm{UU}}=$ 


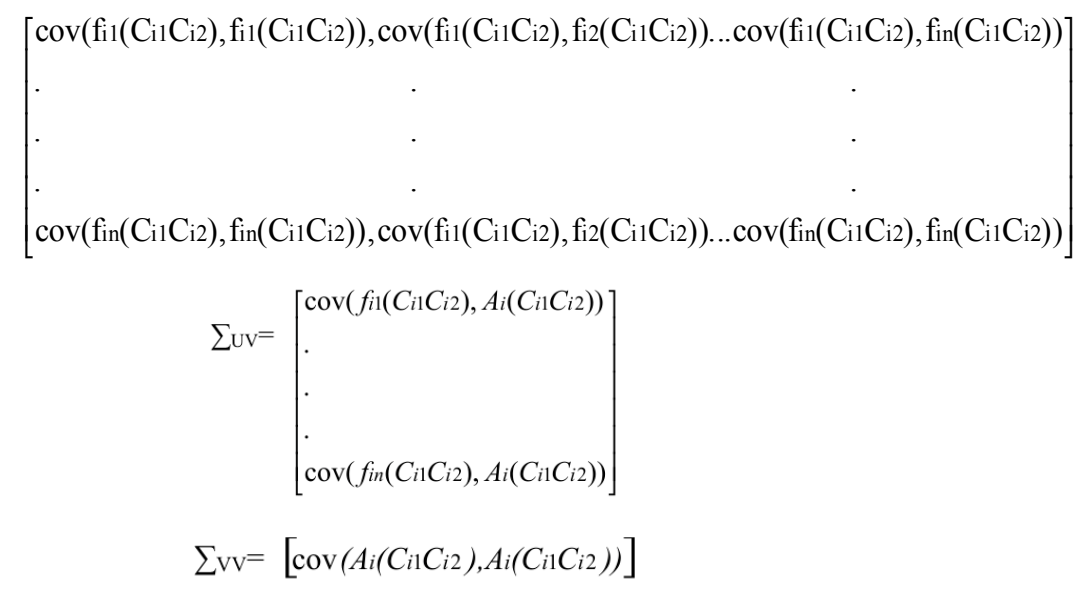

To solve the w that can maximize $\operatorname{corr}\left(\mathrm{w}^{\mathrm{T}} \mathrm{U}, \mathrm{V}\right)$, we use canonical correlation analysis method[9]. The conclusion is that $\mathrm{w}$ is the eigenvector of the largest eigenvalue of the matrix $\Sigma_{\mathrm{UU}}^{-1} \Sigma_{\mathrm{UV}} \Sigma_{\mathrm{VV}}^{-1} \Sigma_{\mathrm{VU}}$.

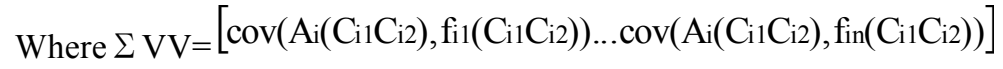

However, the element of $w$ is not the final result of the calculation. Because some elements may be less than 0 , and the sum of remaining elements( greater than 0 ) usually not equal to 1 . The final weight is solved by the following standardized method:

Let $\mathrm{p}=\left(\mathrm{p}_{1}, \mathrm{p}_{2} \ldots \mathrm{p}_{\mathrm{n}}\right)$ is the result of canonical correlation analysis method and $\mathrm{w}=\left[\mathrm{w}_{1}, \mathrm{w}_{2} \ldots \mathrm{w}_{\mathrm{n}}\right]^{\mathrm{T}}$ is the final weights. For all $\mathrm{p}_{\mathrm{l}}<0$, let $\mathrm{p}_{\mathrm{l}}=0$ and $\mathrm{w}_{\mathrm{l}}=0$, then $\mathrm{w}_{1}=\mathrm{p}_{1} /\left(\mathrm{p}_{1}+\mathrm{p}_{2} \ldots \mathrm{p}_{\mathrm{n}}\right)$

\section{Example of Deformed}

We use the 11th stage, the sub-last stage, the last stage rotational blade of a steam turbine as an example to illustrate how to use the above method specifically. In the actual design of bowed and twisted turbine blades, the versatility should be taken into account. It is very helpful to use the type of blade that the factory has been used. Because the workload of designing a bowed and twisted blade is very large, especially the last stage blade is more difficult. The design of a long blade often cost years, so it is an effective way to truncate or lengthen blade to meet the requirement of the design on the basis of the existing blade[10].

The initial work is that two concept pairs are composed of 11th stage, the sub-last stage and the sub-last stage, the last stage. We use $y_{1}, y_{2}, y_{3}$ stand for the 11th stage, the sub-last stage, the last stage rotational blade, respectively. Therefore, we have got two concept pairs $\left(\mathrm{y}_{1}, \mathrm{y}_{2}\right),\left(\mathrm{y}_{2}, \mathrm{y}_{3}\right)$. The specific calculation algorithm is divided into the following three steps. 
First, we should obtain the frequency $f$ and actual semantic similarity of the two sample pairs. As mentioned before, the real frequency value and actual semantic similarity will be replaced by a mean value which is estimated by a certain number of experts. Specifically, six teachers and 30 students who have the knowledge of the design for the blade are invited to carry out this assessment. These teachers and students judge each concept pair on a scale $0,0.1,0.2 \ldots 1$. And then the three highest ones and three lowest ones are removed, so that we get the mean value of the remaining 30 ones. The results is $\mathrm{f}_{1}=0.8125, \mathrm{~A}_{1}=0.76$ and $\mathrm{f}_{2}=0.9331, \mathrm{~A}_{2}=0.9267$

The second step is to calculate the semantic similarity of the function, behavior and structure of the concept pairs. These three similarities can all be calculated by Petrakis's method. For example, with the functional description of $\left(\mathrm{y}_{1}, \mathrm{y}_{2}\right)$ which is mentioned above, we can calculate the semantic similarity . $\mathrm{S}\left(\mathrm{y}_{1}\right) \cap \mathrm{S}\left(\mathrm{y}_{2}\right)=16, \mathrm{~S}\left(\mathrm{y}_{1}\right) \cup$ $\mathrm{S}\left(\mathrm{y}_{2}\right)=22$, based on expression $(2) \operatorname{Sim}_{\mathrm{G}}\left(\mathrm{y}_{1}, \mathrm{y}_{2}\right)=0.7272$. Similarly, $\operatorname{Simx}\left(\mathrm{y}_{1}, \mathrm{y}_{2}\right)$ $=0.6470 . \operatorname{Sim}_{\mathrm{J}}\left(\mathrm{y}_{1}, \mathrm{y}_{2}\right)=0.5238$

Third, calculate the value of the weights and the semantic similarity of the conceptual pairs which is the value of confidence. The results are shown in the following table.

Table 2. semantic similarities of function, behavior and structure, the value of weights

\begin{tabular}{ccccc}
\hline & actual semantic similarity & $\operatorname{Sim}_{\mathrm{G}}$ & $\operatorname{Sim}_{\mathbf{x}}$ & $\mathbf{S i m}_{\mathbf{J}}$ \\
\hline $\mathrm{y}_{1} \mathrm{y}_{2}$ & 0.7600 & 0.7272 & 0.6470 & 0.5238 \\
$\mathrm{y}_{2} \mathrm{y}_{3}$ & 0.8267 & 0.8095 & 0.7500 & 0.6000 \\
$\mathrm{w}$ & & 0.3044 & 0.6956 & 0 \\
\hline
\end{tabular}

According to the weights and semantic similarity based on expression (1), we can get $\mathrm{c}\left(\mathrm{y}_{1}, \mathrm{y}_{2}\right)=0.6715, \mathrm{c}\left(\mathrm{y}_{2}, \mathrm{y}_{3}\right)=0.7681$. In the non-axiomatic design logic, we can express the above deformation process as a graph.

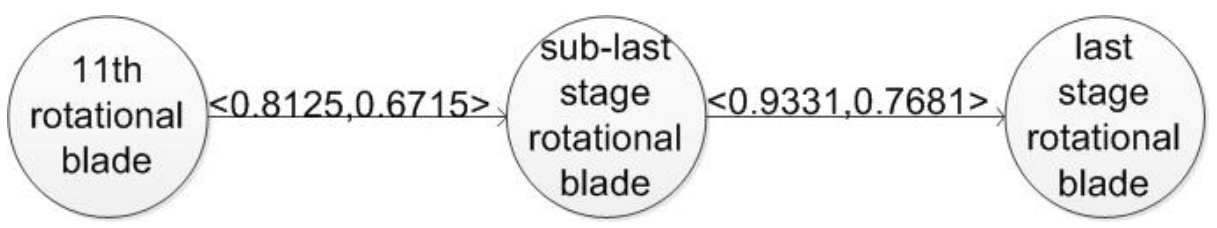

Fig. 2. An example of propositions

For some reason (for example, we want to optimize the design of the last blade), we may wish to directly deform from the 11 rotational blades to the last rotational blades. This is a process of deduction. With the conditions given and the calculation method mentioned in Table 1 , the true value of (11th rotational blade $\rightarrow$ last stage rotational blade) is $<0.7581,0.3910>$. 


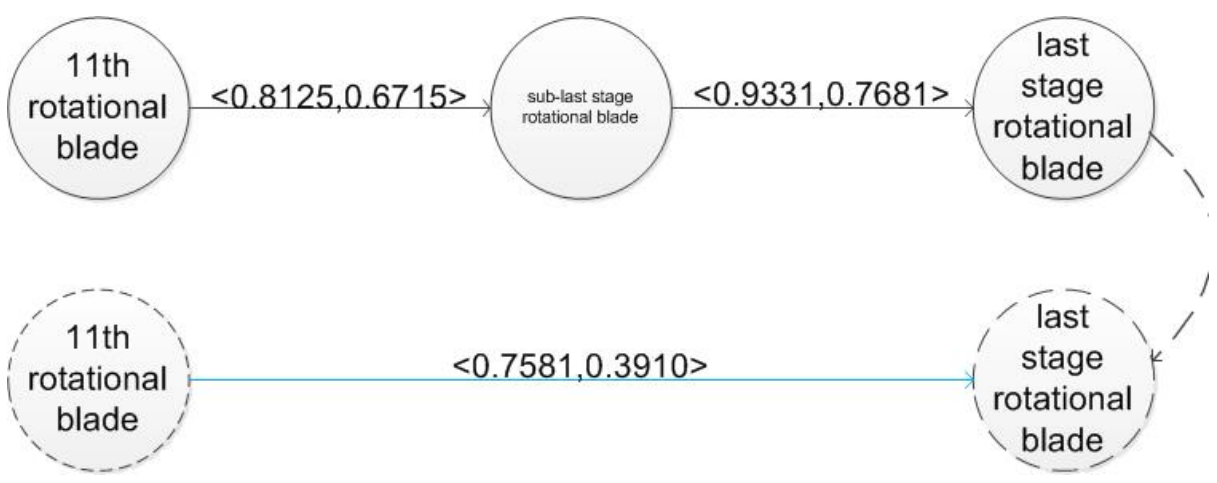

Fig. 3. A deductive deformation design reasoning

\section{Conclusion}

In this paper, the non-axiom logic is applied to the deformation design, and the blades of steam turbine are taken as an example to prove that this method is feasible. The main innovation is that the SBF model and an algorithm for confidence are added to the non-axiom logic which effectively improves the accuracy of true value of the non-axiom logic. The method can achieve a quantitative assessment of the deformation process. With insufficient knowledge, the goals of getting rid of the heavy repetitive work of conventional design and making the product more innovative have been realized. In the process of practical application of NAL, we also found some characteristics of this theory.

\section{$7 \quad$ Acknowledgment}

Funding supported by National natrual Science Foundation of China (No $51365010)$.

\section{$8 \quad$ References}

[1] Bhatta, S. R. , \& Goel, A. K. (1997). A functional theory of design patterns. International Joint Conference on Artifical Intelligence (Vol. 55, pp. 294-300). Morgan Kaufmann Publishers Inc.

[2] Prabhakar S, Goel A K. (1998). Functional modeling for enabling adaptive design of devices for new environments. Artificial Intelligence in Engineering, 12(4):417-444. https://doi.org/10.1016/S0954-1810(98)00003-X

[3] Wang, P. (2011). Rigid Flexibility: The Logic of Intelligence. Springer Publishing Company, Incorporated.

[4] Kazakçı, A. O. , Hatchuel, A. , \& Weil, B. (2008). A model of ck design theory based on term logic: a formal ck background for a class of design assistants. Lamsade. dauphine. fr. 
[5] Chen Y, Huang J, Xie Y, et al. (2013) Modeling detailed design knowledge with the extended structure-behavior-function model. Artificial Intelligence for Engineering Design Analysis \& Manufacturing, 27(4):415-420. https://doi.org/10.1017/S0890060413000164

[6] Patil, L. , Dutta, D. , \& Sriram, R. (2005). Ontology-based exchange of product data semantics. IEEE Transactions on Automation Science \& Engineering, 2(3): 213-225. https://doi.org/10.1109/TASE.2005.849087

[7] Abdul-Ghafour, S. , Ghodous, P. , Shariat, B. , Perna, E. , \& Khosrowshahi, F. (2014). Semantic interoperability of knowledge in feature-based cad models. Computer-Aided Design, 56(11):45-57. https://doi.org/10.1016/j.cad.2014.06.001

[8] Lu W, Qin Y, Qi Q, et al (2016). Selecting a semantic similarity measure for concepts in two different CAD model data ontologies[J]. Advanced Engineering Informatics, 30(3):449-466. https://doi.org/10.1016/j.aei.2016.06.001

[9] Härdle, W. K. , \& Hlávka, Z. (2012). Canonical correlation analysis. Journal of Financial Economic Policy, 6(2):179-196. https://doi.org/10.1007/978-3-642-17229-8 15

[10] Shi Zhoudao. (1990) . Turbine Design Basics. Beijing: China Machine Press, pp. 90

\section{Authors}

Qinzhou Niu is a professor of college of Information Science and Technology in the Guilin University of Technology. He mainly works in the area of manufacturing informatization and artificial intelligence.

Yi Liu is with the College of Mechanical and Control Engineering, Guilin University of Technology, Guilin, China.

Article submitted 11 February 2018. Final acceptance 05 March 2018. Final version published as submitted by the authors. 